\title{
Practice projects in optics for high school students of the Kolmogorov School of Moscow State University
}

\section{Valentin Lobyshev, A. Golubkov}

Valentin I. Lobyshev, A. A. Golubkov, "Practice projects in optics for high school students of the Kolmogorov School of Moscow State University," Proc.

SPIE 2525, 1995 International Conference on Education in Optics, (13 October 1995); doi: 10.1117/12.224065

Event: SPIE's 1995 International Symposium on Optical Science, Engineering, and Instrumentation, 1995, San Diego, CA, United States 
Practice projects in optics for high school students

of the Kolmogorov school of Moscow State University

V.I.Lobyshev, A.A.Golubkov

Moscow State University, Kolmogorov school

Kremenchugskaya 11, Moscow 121357, Russia

\begin{abstract}
The description of the optic laboratory including the projects based on the laser optical complex in Kolmogorov high school is given
\end{abstract}

\title{
INTRODUCTION
}

Moscow State University high school named after the well known mathematician A.N.Kolmogorov was founded in 1963 as a physics and mathematics school for the gifted students of 14 to 16 . Using the multiple level entrance exams the best students were chosen from the European part of the former USSR, now from Russia. Two types of courses, one and two year, are available. The students have 8 hours of physics a week which includes 2 hours of lectures, 4 hours of seminars and 2 hours of laboratory. Close attention is paid to the scientific research work of students outside the class. The level of students is traditionally high and practically all of them enter Moscow State University or other universities of Moscow.

The optic laboratory is a part of the general physics laboratory. Eight projects are suggested for the two year course. The projects of different complexity level are made sequentially starting from the easiest and ending with those which demand high experience. Preparing for lab, the student reads the description of the problem and prepares to answer basic theoretical questions, After answering these questions the student is allowed to make the lab work during two hours. At the next lesson the experimental results must be presented and defended. During a week in between the student studies the problem more thoroughly, makes necessary calculations and estimates the accuracy of the results.

\section{FORMULATION OF THE PROBLEMS}

The whole list of optical problems is as follows:

1. Determination of the focal lengths of spherical mirrors and lenses.

2. Principles of the microscopy.

3. Graduation of the spectroscope and determination of the wavelengths in the spectra of gases.

4. Study of the illuminance laws using a semiconductor photoelectric cell.

5. Determination of wavelengths in optical spectra using the diffraction grating.

6. Study of distribution of light intensity in a laser beam.

7. Study of diffraction phenomena. Fresnel and Fraunhofer diffraction.

8. Study of interference of light. The experiment of T.Young. 


\section{DESCRIPTION OF THE PROBLEMS}

Problem 1 uses optical bench with the ruler, screen, filament lamp as a source of light and the set of lenses and mirrors. The construction of a microscope is investigated in problem 2 . These two problems are rather simple and does not demand any knowledge beyond the ordinary school syllabus. The subsequent problems are more complex.

In problem 3 the prism spectroscope, high voltage source and the set of gaseous spectral tubes / Geissler tubes / are used. The students start with acquaintance with the spectroscope and then obtain its graduation curve using the known spectral lines of helium. Then they use this curve to study the spectra of several gases / hydrogen, neon, krypton / and compare the results with the data from the handbook. During the concluding discussion with the teacher the questions concerning the types of spectra and their nature, the construction of the spectroscope, dispersion of light are highlighted.

The aim of problem 4 is the acquaintance with the basic principles of a selenium photoelectric cell and the experimental study of the illuminance laws. Students get additional information about the mechanism of functioning of a semiconductor photoelectric cell. The dependence of photocurrent on the intensity of luminous flux is obtained. The dependence of illuminance against the distance from the light source as well as against the falling angle is also studied. The results obtained are compared with the theory. In the concluding discussion the main attention is paid to the analysis of the results obtained and their consistency with the theory.

In problem 5 a precise quantitative study of diffraction phenomena is carried out using simple equipment: the incandescent lamp, diffraction grating, measuring panel and the ruler with the slit. Students get acquainted with the construction and functioning of the diffraction grating. Studying the diffraction spectrum they determine the wavelength of red, yellow, blue and violet light. In order to improve the quality of measurements the corresponding light filters may be used. In the concluding discussion the principles of diffraction phenomena, characteristics of diffraction grating, it's resolution and the role of diffraction phenomena in optical measurements are highlighted.

The last three problems use a compact universal laser optical complex consisting of the $\mathrm{He}-\mathrm{Ne}$ laser, optical bench, photoelectric unit with registering voltmeter and the large number of adjusting optical modules including lenses, polarizers and modules for changeable objects such as calibrated grid, several slits. holes, disks, plates, prisms, lenses. The construction of the modules allows to drop them occasionally without damage. This feature is very useful for inexperienced students and maybe even more for their teachers.

Problem 6 is devoted to the acquaintance with the optical complex and study of laser beam characteristics. The students start investigating with the transverse distribution of light intensity of the initial, unperturbed laser beam at several distances from the source. The students check its "Gausslike" shape and calculate the linear and spatial angle of divergence of the beam. Then the dependence of light intensity in a spherical wave, created by lens on the distance from the point source or the focal point of the beam is studied. Using the results obtained the location of the focal point of the

laser beam is calculated and the focus of the lens is determined. After that the focal length is 
determined by traditional methods of geometrical optics. The precision, area of applicability, advantages and disadvantages of the methods used for measuring focal lengths are discussed.

Problem 7 also uses the laser optical complex and is devoted to investigation of the phenomena related to Fresnel and Fraunhofer diffraction. The wavelength of laser radiation is determined and several theoretical predictions are checked. In particular the dependence of light intensity in the middle of the beam on the distance from the hole and parameters of the Fresnels spiral are determined. The validity of the Babinet principle is also checked in the work. The Huygens Fresnel principle, the zone theory of Fresnel, the features of Fraunhofer diffraction and the Babinet principle are the main points of discussion.

In problem 8 the observation and study of the interference phenomena within the Young experiment is carried out, the distribution of intensity in the interference picture is measured and the wavelength of laser beam is determined. The principal feature of this work is that it is formulated as a brief plan only, and the students have to work out the details of their own experiment. The main attention is paid to the justification of the suggested plan of experiment, the explanation of results obtained. The concepts of coherence, coherent light waves, time and length of coherence are discussed.

Laser optical complex allows to perform much more experiments. The most curious students have an opportunity to enlarge their knowledge and experience in experimental physics after the lessons. We continuously improve our laboratory and try to make it more interesting for students. Two new problems, "Light and color in nature" and "Holography" are forthcoming.

E.mail: lob@school.phys.msu.su

FAX: 1007-095/445-4634 